\title{
FDLA: A Novel Frequency Diversity and Link Aggregation Solution for Handover in an Indoor Vehicular VLC Network
}

\author{
Elnaz Alizadeh Jarchlo Member, IEEE, Elizabeth Eso Member, IEEE, Hossein Doroud, \\ Anatolij Zubow Member, IEEE, Falko Dressler Fellow, IEEE, Zabih Ghassemlooy Senior Member, IEEE, \\ Bernhard Siessegger Member, IEEE, Giuseppe Caire Fellow, IEEE
}

\begin{abstract}
Visible light communications (VLC) (VLC) has been introduced as a complementary wireless technology that can be widely used in industrial indoor environments where automated guided vehicles aim to ease and accelerate logistics. Despite its advantages, there is one significant drawback of using an indoor vehicular VLC (V-VLC) network that is there is a high handover outage duration. In line-of-sight VLC links, such handovers are frequently due to mobility, shadowing, and obstacles. In this paper, we propose a frequency diversity and link aggregation solution, which is a novel technique in Data link layer to tackle handover challenge in indoor V-VLC networks. We have developed a smallscale prototype and experimentally evaluated its performance for a variety of scenarios and compared the results with other handover techniques. We also assessed the configuration options in more detail, in particular focusing on different network traffic types and various address resolution protocol intervals. The measurement results demonstrate the advantages of our approach for lowoutage duration handovers in V-VLC. The proposed idea is able to decrease the handover outage duration in a two-dimensional network to about $0.2 \mathrm{~s}$, which is considerably lower compared to previous solutions.
\end{abstract}

Index Terms-Visible Light Communication; Handover; Outage Duration; Link Aggregation; Frequency Diversity; Visible Light Sensors; VLC Data Link Layer

\section{INTRODUCTION}

$\mathbf{V}$ ISIBLE light communications (VLC) is a promising wireless technology for indoor data communications. Visible light sensors (VLS) can act as light access points (LAP) or light clients (LC). Given that, LAPs have limited wireless coverage, a dense grid of LAPs is required to provide a seamless wireless coverage in vehicular VLC (V-VLC) networks. V-VLC is very robust against jamming attacks, offers a smaller interference domain and uses a large spectrum for vehicular communication networks [1]. In V-VLC, vehicles are required to have access to real-time information including possible collisions, congestion, traffic signal violations, emergency brakes and locations [1], [2]. This information can be used to monitor the traffic and

This work was supported in part by European project called VisIoN, funded by the European Unions Horizon 2020 research and innovation program under the Marie Sklodowska-Curie grant agreement number 764461 .

E. Alizadeh Jarchlo, H. Doroud, A. Zubow, F. Dressler, and G. Caire are with School of Electrical Engineering and Computer Science, TU Berlin, Germany, e-mail: \{alizadeh,doroud,zubow,dressler\}@tkn.tu-berlin.de, caire@tu-berlin.de,

E. Eso, Z. Ghassemlooy are with Optical Communication Research Group, Northumbria University, UK, e-mail: \{elizabeth.eso,z.ghassemlooy\}@northumbria.ac.uk,

B.Siessegger, b.siessegger@web.de improve energy and cost efficiencies within indoor and outdoor environments. Currently, technologies that could be adopted for vehicle to infrastructure (V2I) communications are in their early stages and researchers are exploring V-VLC networks and Data link layer, which are offering new challenges.

Research challenges include, for example, the MAC and upper layers V-VLC. V-VLC systems are required to offer user mobility with handover capabilities to maintain seamless communications, which needs addressing [3], [4]. In V-VLC -based networks, with many LAPs and the need for handover management it is essential to ensure connectivity between mobile users (MUs). Therefore, the need for measuring the network responsiveness under varying degrees of interference and the LAP backhaul traffic overhead due to the control channels, which occur only with mobility [3], [4]. Moreover, blocking, spatial diversity, and access to the channel are also considered in VLC-based channel access mechanism for indoor communications, which have been reported in the literature [5], [6]. However, studies on inter-vehicles communications are relatively sparse [7]. Note, in vehicular communications high reliability and low outage durations are essential requirements, which can be addressed using the VLC technology [8].

In this paper, we focus on the handover in V-VLC and address challenges it introduces under mobility with the aim of reducing the handover outage duration. Our objective is to decrease handover outage duration ( $\left.T_{\text {hod }}\right)$ in an indoor V-VLC network environment. To increase VLC network coverage and reduce the interference and signal distortion levels, one effective option would be to adopt frequency diversity by having more than one LC on an automated guided vehicles (AGV) with more than one LAP operating at different frequency bands.

Note, AGV may use two different frequency bands to establish communication links when within the range of a new LAP. However, the link connection with LAPs may be lost when AGV makes sudden change of directions. To address this problem, in this work we proposed a frequency diversity and link aggregation solution (FDLA) by assigning 3-LC per $\mathrm{AGV}$, where each LC is assigned a unique frequency range that is compatible with its corresponding LAP. Thus, ensuring link availability for AGV when moving in all directions. In implementing this we need to address the followings: $(i)$ when and how to switch between VLS interfaces for mobile AGVs with a minimum delay time during the handover procedure; and (ii) how to choose the best available VLC link when there 
is more than one available.

In this paper, which is built upon our previous work in [8], we revisit and extend the architectural design to enable V-VLC to decrease handover delay in two-dimensional (2D) in indoor industrial environments. We configure each pair of VLSs to establish links using one of the three predefined frequency bands and aggregate Ethernet links on the client side. Therefore, in a V-VLC network and at any given time and direction, we select the best link established between the client and the server in both up- and down-link with the minimum handover delay times. We investigate and evaluate the performance of the proposed FDLA system using three different use case scenarios and traffic types to gain a better understanding of the mobility and handover in V-VLC networks.

The remainder of this paper is organized as follows: Section II briefly gives an overview of the existing related approaches and Section III details the potential problems, which arise due to handover in a V-VLC network, and the motivation for the proposed FDLA system. Section IV presents the proposed solution for handover in details. Section $\mathrm{V}$ describes three different V-VLC network scenarios and Section VI describes the approaches adopted in designing the system architecture in detail. Section VII demonstrates the implementation of the algorithm in real-world case studies. Finally, Section VIII concludes the paper.

\section{RELATED WORK}

A growing number of research works on the handover in VLC and wireless mobile networks have been reported in the literature, which are mostly focused on proposing schemes to achieve a smooth handover with decreasing data rate fluctuation and increasing transmission bandwidth [9], [10]. In addition, in [8], [11] and [12], we proposed and evaluated new architectural designs, aiming at handover delay decrement and VLC network throughput increment.

\section{A. Handover techniques in wireless networks}

There are several existing handover techniques using wireless technologies which aimed to provide a seamless handover like FDLA in mobile networks. In [13], mobility in VLC were investigated and a simulation-based handover approach was proposed to achieve seamless connectivity for two different indoor scenarios consisting of non-overlapping and overlapping lightings based on the received signal intensities. The proposed scheme was based on the buffer size adjustment, initiation of handover before disconnection and establishing new connections with the neighboring cell prior to leaving the serving light cell. Moreover, the statistical distribution of received data rates by means of simulations as well as frequency- and power-based soft handover methods were proposed in [9] for reducing data rate fluctuations as MUs moving between cells. In [14], an optimal Lambertian order and a handover algorithm were proposed to increase the transmission bandwidth in a VLC system by reducing the multipath induced dispersion. This was achieved using an algorithm, where in a multicell network cells not covering the MU were deactivated to decrease the overall root mean square delay spread. Also, in [10], a handover procedure was proposed based on a pre-handover scheme, which was based on the position estimation using visible light positioning and motion tracking with Kalman filters.

In [15], a hybrid communications system supporting vertical handover between radio frequency (RF) and VLC links was proposed, where with no access to the primary VLC link the RF link was used. To ensure efficient handover, link monitoring and decision-making schemes were defined between the Data link and the network layer.

In [16], a cognitive indoor VLC system using multiple access points (APs), which served primary and secondary users using orthogonal frequency division multiple access (OFDMA), was presented. The OFDMA-based network was defined in terms of the physical area and the number of allocated subcarriers for the regions of primary and secondary MUs. A light-based cell design with cognitive constraints was proposed to assure sufficient illumination, handover, and mobility requirements. Consequently, a realistic scenario was investigated to evaluate the performance of the proposed scheme using Monte Carlo simulations. The results obtained showed that, using the optimum value for the mobility parameter the desired requirements could be realized, while still attaining within the cell a high level of spectral efficiency.

In [17], an algorithm called BIGAP was proposed with the purpose of providing both improved network performance and seamless mobility in the RF domain. The proposed algorithm was designed based on various channel assignment to the colocated APs using the available RF spectrum. Note, dynamic selection of the operating frequencies was carried out by BIGAP, which is compatible with IEEE-802.11, forcing the client to change APs. BIGAP improved $T_{\text {hod }}$ significantly with frequent and seamless handover supporting efficient load balancing within the network. This approach is similar to FDLA, since both use different channel frequencies. In contrast to BIGAP, where there is the need for a single network interface, FDLA requires multiple VLC interfaces.

In this work, we use prototype devices provided by OSRAM, where neighboring LAPs and clients are assigned different frequencies similar to [16] and a logical bond interface on the client side is adopted to select the VLC link any time as part of the VLC network. In FDLA link selectivity is based on the speed and the availability of duplex links with the aim of improving $T_{\text {hod }}$ during handover and maintaining the highest data rates in the V-VLC network. FDLA is designed to work in an indoor environment similar to handover technique introduced in [13] and it provides continues link monitoring to detect the VLC link failure as early as possible like conducted in [15].

\section{B. Comparison to the state of the art}

Note that to the best of authors knowledge this paper is the first to evaluate $T_{\text {hod }}$ in a V-VLC network with two dimensions movements, considering possible scenarios where an AGV experiences link failure during handover. Previously, in [11] and [8], we initially proposed the idea of using link aggregation on top of multiple VLC clients for AGV and frequency division 


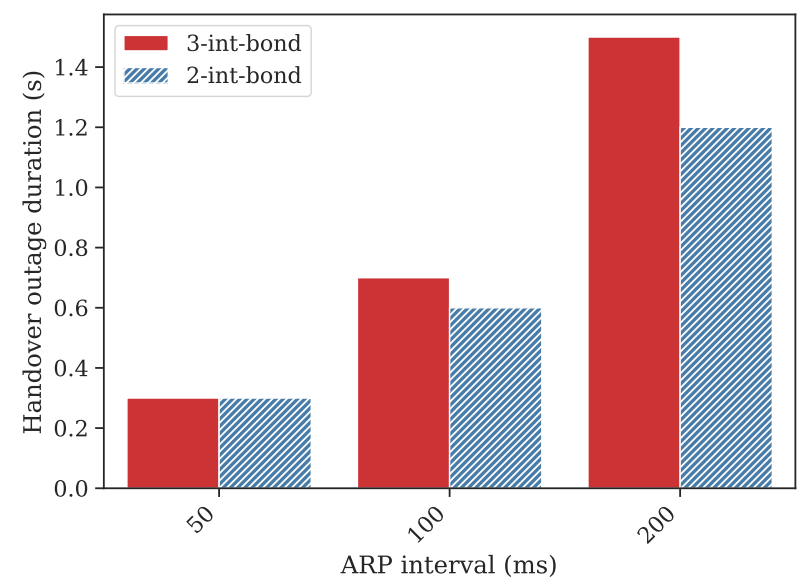

Fig. 1. Comparison of TCP handover outage duration for the cases with two and three primary interfaces.

for neighboring LAPs and their corresponding LCs. In addition, we analyzed an interface bonding scheme using only two different primary interfaces and evaluated $T_{\text {hod }}$ for the AGV with a linear movement [8], [11].

Fig. 1 and 2 present comparisons of achieved $T_{\text {hod }}$ for the V-VLCs network cases using two and three primary interfaces for transmission control protocol (TCP) and User Datagram Protocol (UDP) packet transmissions, respectively. As it is presented the values of $T_{\text {hod }}$ for the case with only two primary interfaces, are slightly lower than the case where an AGV client is equipped with three VLC interfaces in most of cases. The reason for this is that detecting the available link to switch will take longer for the case where the number of interfaces are more than two. However, the achieved $T_{\text {hod }}$ is still much lower compared with the case where there is no handover technique is used, see Section III.

Moreover, in [12], we applied multipath-transmission control protocol (MPTCP) for a V-VLC network to improve the network performance in terms of the network outage duration and throughout. We considered the transport layer of VLC with the two different MPTCP schedulers; Default and Redundant, and also Backup MPTCP tool to investigate their impact on the VLC performance under mobility (i.e., handover) and shadowing/obstacle. We presented a real-world experiment using the same VLC devices to demonstrate the improvement in the network throughput and outage duration depends on the user case scenarios with frequent handovers. As it is presented in Table I, the achieved $T_{\text {hod }}$ using Redundant scheduler in MPTCP is $0.02 \mathrm{~s}$, due to broadcasting the traffic over all available sub-flows regardless of path's characteristics.

TABLE I

COMPARISON OF $T_{\text {hod }}$ USING MPTCP AND LINK AGGREGATION METHODS.

\begin{tabular}{lllllll}
\hline & \multicolumn{3}{c}{ MPTCP } & \multicolumn{3}{c}{ Link Aggregation } \\
$\begin{array}{l}\text { Schedulers/ } \\
\text { ARP int }(\mathrm{ms})\end{array}$ & Redundant & Default & Backup & $\mathbf{5 0}$ & $\mathbf{1 0 0}$ & $\mathbf{2 0 0}$ \\
\hline$T_{\mathrm{hod}}(\mathrm{s})$ & 0.02 & 0.3 & 0.6 & 0.3 & 0.7 & 1.5 \\
\hline
\end{tabular}



Fig. 2. Comparison of UDP handover outage duration for the cases with two and three primary interfaces.

However, the result shows a trade-off between bandwidth utilization and latency [12]. Although using link aggregation in Data link layer leads to a higher value for $T_{\text {hod }}$, it improves the network throughput at the same time [12]. As it is shown in Table I that $T_{\text {hod }}$ using link aggregation with an address resolution protocol (ARP) interval of $50 \mathrm{~ms}$ is $0.3 \mathrm{~s}$, which is lower than using the MPTCP backup mode where in case of any of the sub-flows failure, the backup interface is selected as the backup for establishing TCP. In principle, the MPTCP backup tool and the link aggregation method work quite similarly except for ARP monitoring tool and a backup link, which is selected based on the speed and duplexity.

\section{Problem Statement}

VLC networks provide secure and high data rate connectivity in indoor applications where there is a high demand for network reliability with low handover delay, i.e., low $T_{\text {hod }}$. However, in situations with a high degree of mobility the network experience frequent handovers between the LAPs due to shadowing (i.e., blind spots) and blocking particularly in VLC. In indoor industrial environments with many LAPs, data transmission between LC and a remote server situated far from the V-VLC network may experience link failures due to ongoing handovers between the LCs and LAPs

In some cases, $T_{\text {hod }}$ can be as high as tens of seconds, which is not desirable in VLC networks. To address this and deal with high mobility, one possible solution would be to adopt frequency diversity to avoid channel access conflicts during handover, where the receivers (Rxs) can receive signals on multiple channels with different carrier frequencies.

For the system with frequency diversity, we should assign unique frequencies to (i) two LCs per AGV each with its; and (ii) LAPs installed on the ceiling to establish links with the corresponding available LCs. In this work, we have emulated frequency division multiple access (FDMA) using two pairs of LSs and repeated the experiment 10 times. As depicted in Fig. 3, for UDP packet transmission results show that, the average $T_{\text {hod }}$ for FDMA is $3.9 \mathrm{~s}$, which is lower by $11 \mathrm{~s}$ compared with no handover. This is due to the fact that, following a link 




Fig. 3. Outage duration for selected VLC handover techniques.

failure it will take a couple of seconds for the LC to monitor the existing channels and establish a new link using a free channel.

For FDLA we have shown two $T_{\text {hod }}$ values as 3 and $0.2 \mathrm{~s}$ for ARP intervals 200 and $50 \mathrm{~ms}$ respectively, see Fig. 3. As it is presented, the achieved values are much lower than the case with no handover (more on this in section VII).

\section{COMmunication System And Network ARCHITECTURE}

In this section, we introduce the designed V-VLC system and the potential network architecture. The latter include an AGV with 3-LS. Higher number of LSs can be used to increase the coverage area, however, three is the minimum number that can support 2D movements of vehicles. Note, each LS in AGV acts as a LC with assigned non-overlapped frequency bands, which can establish a link with their corresponding LAP.

Fig. 4 shows a possible network architecture with 3 cells (i.e., 3-LAP) and a AGV with 3-LC. For the links between $\mathrm{LC}_{1}-\mathrm{LAP}_{1}, \mathrm{LC}_{2}-\mathrm{LAP}_{2}$ and $\mathrm{LC}_{3}-\mathrm{LAP}_{3}$ we have assigned the frequencies of $F_{1}, F_{2}$ and $F_{3}$, respectively, see Table II for the values. Care has been taken in assigning the frequencies to avoid inter-link interference. As shown in Fig. 4 using three LAPs will results in overlapping areas of $A_{x}$ and $A_{y}$ to allow handover. Note, LAPs are connected to the server via a switch. Each AGV is equipped with a mini-PC and an indoor VLC mobile client system, which is described in following subsections.

The LSs used are COTS VLC devices provided by OSRAM, which uses OFDM and G.hn standard to provide an average real-time bidirectional communication at $90 \mathrm{Mbps}$ over a transmission distance of $10 \mathrm{~m}$ [17]. LSs have five off-theshelf high-power LEDs (OSRAM SFH 4715 AS) with 60 degree half angles and four large-area silicon PDs (Hamamatsu S6968). The transmit power of LEDs and their effective areas are $630 \mathrm{~mW}$ and $150 \mathrm{~mm}^{2}$, respectively.

In this work, we consider Indoor Mobile Client (IMC) with 3-LS connected via the standard Ethernet interface as shown in Fig. 5. This novel architecture uses no custom-made software/hardware module at neither in IMC or in LC and the



Fig. 4. Flight network architecture.

TABLE II

EXPERIMENTAL PARAMETERS

\begin{tabular}{ll}
\hline Parameter & Value \\
\hline Frequency band $F_{1}$ & $2-29 \mathrm{MHz}$ \\
Frequency band $F_{2}$ & $29-59 \mathrm{MHz}$ \\
Frequency band $F_{3}$ & $59-94 \mathrm{MHz}$ \\
Distance between LCs and LAPs & $2 \mathrm{~m}$ \\
Link blockage duration time for shadowing/blocking & $3 \mathrm{~s}$ \\
PD's field of view (FoV) & 60 degree \\
Average throughput between LAP and LC & $90 \mathrm{Mbps}$ \\
Room dimension & $5 \times 4 \times 2.7 \mathrm{~m}^{3}$ \\
Distance between LAPs & $1 \mathrm{~m}$ \\
VLC channel Bandwidth & $100 \mathrm{MHz}$ \\
Transmission power of LED & $630 \mathrm{~mW}$ \\
Effective active area of PD & $150 \mathrm{~mm}$ \\
Number of LEDs in a VLC COTS & 5 \\
Number of PDs in a VLC COTS & 4 \\
\hline
\end{tabular}

IMC already has multiple standard wired network interfaces (i.e., Ethernet) for linking IMC's output to the next network hop (i.e., switch or router), thus facilitating VLC.

\section{Definition of Three Use-Case Scenarios}

In this section, we consider three critical scenarios, see Fig. 6, where an AGV may experience link loss during handover and outline FDLA for addressing the problem due to handover.

\section{A. Scenario 1: A single VLC link}

For the case 1 in Fig. 6, the AGV is moving along a linear path in a given direction with LAPs continue providing

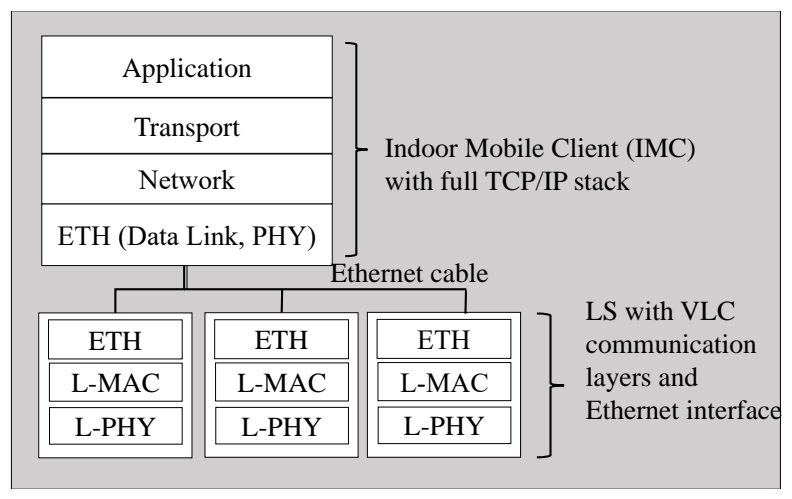

Fig. 5. Indoor VLC mobile client diagram. 


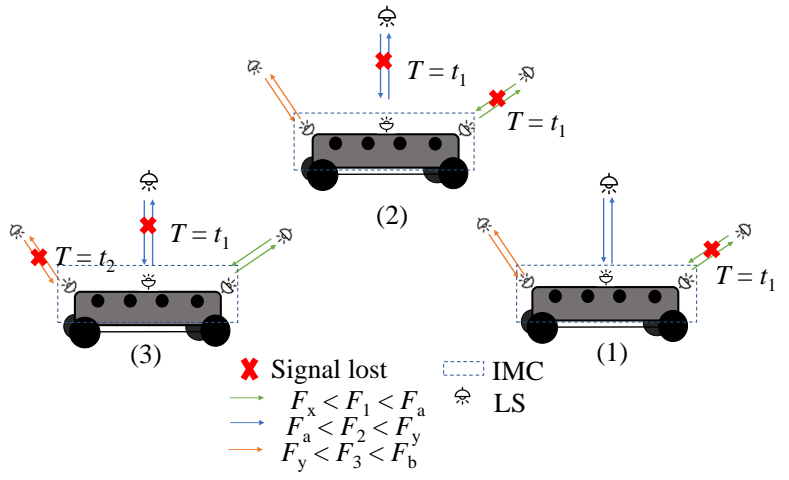

Fig. 6. Three Different scenarios: (1), (2) and (3) present scenario 1 with a single link broken at $\mathrm{T}=\mathrm{t} 1$, scenario 2 with two links broken at $\mathrm{T}=\mathrm{t} 1$ and scenario 3 with two links broken at $\mathrm{T}=\mathrm{t} 1$ and $\mathrm{T}=\mathrm{t} 2$, respectively.

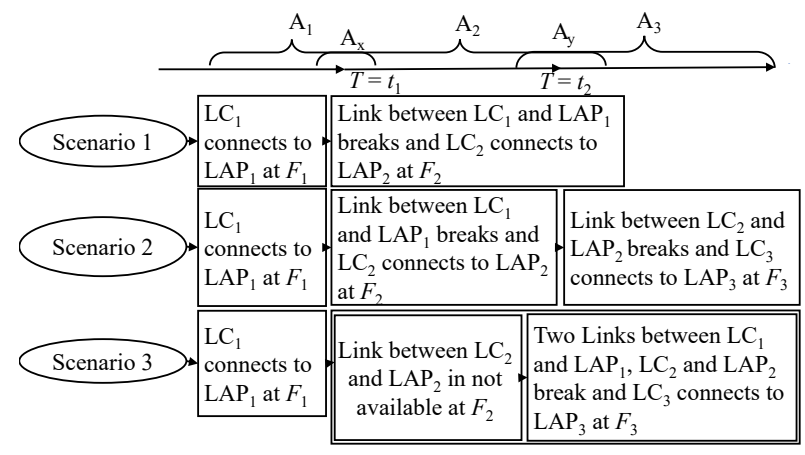

Fig. 7. Flowcharts for three different scenarios.

seamless coverage. Note, handover between IMC and LAPs will takes place when IMC between cells i.e., coverage areas, e.g., $\mathrm{A}_{1}$ and $\mathrm{A}_{2}$, see Fig. 7. With $\mathrm{AGV}$ entering $\mathrm{A}_{1} T=t_{1}$, $\mathrm{LC}_{1}$ is connected to $\mathrm{LAP}_{1}$ with $F_{1}$ and when it is at $\mathrm{A}_{x}$ there will be two parallel VLC active links between the LCs and corresponding LAPs. With AGV continue moving towards $\mathrm{A}_{2}$, the link between $\mathrm{LC}_{1}$ and $\mathrm{LAP}_{1}$ is lost and only the link between $\mathrm{LC}_{2}$ and $\mathrm{LAP}_{2}$ with $F_{2}$ stays active.

\section{B. Scenario 2: Lose of two VLC link connections concurrently.}

For case 2 in Fig. 6, AGV has no access to two VLC links due to shadowing, blocking, etc. In this case, shown in scenario 2 in Fig. 7, an AGV enters $A_{2}$ at $T=t_{1}$ and loses the connection with $\mathrm{LAP}_{1}$. However, due to obstacle or shadowing, the link with $\mathrm{LAP}_{2}$ is not available too. Therefore, arriving at $\mathrm{A}_{3}$ coverage areas the IMC establishes a link connection between $\mathrm{LC}_{3}$ and $\mathrm{LAP}_{3}$ on $F_{3}$.

\section{Scenario 3: Change of direction and lose of two VLC link connections, respectively.}

For case 3 in Fig. 6, the AGV way lose the two VLC links at $t_{1}$ and $t_{2}$, therefore it is moving from $\mathrm{A}_{1}$ to $\mathrm{A}_{3}$ via $\mathrm{A}_{2}$ as shown in Fig. 7. At first, AGV enters and the same happens as scenario 1 until it arrives by $\mathrm{A}_{2}$ coverage area at $T=t_{1}$. Therefore, there is a VLC link connection established between $\mathrm{LC}_{2}$ and $\mathrm{LAP}_{2}$ on $F_{2}$ frequency band and it continues towards $\mathrm{A}_{3}$. Before the AGV enters the $\mathrm{A}_{3}$ area at $T=t_{2}$, there will be two parallel VLC active links between the LCs and corresponding LAPs. Eventually, when the AGV keeps riding towards to $\mathrm{A}_{3}$ area, the link between $\mathrm{LC}_{2}$ and $\mathrm{LAP}_{2}$ breaks and only the link between $\mathrm{LC}_{3}$ and $\mathrm{LAP}_{3}$ on $F_{3}$ frequency band remains active.

\section{FDLA System CONCEPT}

FDLA is a flexible network architecture based on frequency diversity (FD) and link aggregation methods and is used for the first time in a 2D V-VLC network environment. It is proposed to deal with handover related problems by decreasing $T_{\text {hod }}$ and improving network reliability, robustness, and coverage under different use-case scenarios.

\section{A. Frequency diversity}

Using FD, which is based on FDMA per node with three nonoverlapping frequency bands, the coverage area of 3-LSs can be extended and link availability can be improved under shadowing, blocking, mobility and handover.

Each pair of LAP and its corresponding LC are assigned with non-overlapping frequency bands of $F_{1}, F_{2}$ and $F_{3}$. On the IMC side, $\mathrm{LC}_{1}$ is configured with $F_{1}$ and $\mathrm{LC}_{2}$ is configured with $F_{2}$. Therefore, there is an aerial view of LAP coverage plan provides a seamless VLC coverage for the IMC mobility scenarios with only three non-overlapping frequency groups $\left(F_{1}, F_{2}\right.$, and $\left.F_{3}\right)$.

\section{B. Link aggregation}

To decrease $T_{\text {hod }}$, link aggregation (network bonding) is implemented at Ethernet interface of the IMC's Data link layer within the system installed on AGV by combining parallel network connections into a single logical interface called a bond interface (BI). There are seven different types of network bonding modes, ${ }^{1}$ which are briefly explained below (see also [8]).

- Mode 0 - balance-rr: In this mode, packets are transmitted in a sequential order from the first available slave through to the last.

- Mode 1 - active-backup: Only one of the available slave interfaces is active at any one time. Therefore, on failure of the active slave the second slave will be activated

- Mode 2 - XOR: XOR: Bit-wise XOR operation on the source and destination MAC addresses are carried out during packet transmission.

- Mode 3 - broadcast: The same packet is transmitted over all slave interfaces.

- Mode 4 - 802.3ad: Creates aggregation groups, which share the same speed and duplex settings, that utilizes all slaves in the active aggregator based on 802.3ad specifications.

- Mode 5 - balance-tlb: The traffic is distributed based on the current load per slave. In the case of current slave failure, another slave is used for the MAC address of the failed receiving slave.

${ }^{1} \mathrm{http} / / /$ knowledgebase.45drives.com/kb/implementing-network-bonding-oncentos-7/ 
- Mode 6 - balance-alb: The received load balancing is achieved using ARP. The bonding driver intercepts ARP acknowledgement message sent by the local system and over-writes the source MAC address with the unique MAC address of one of the slaves in the bond. Thus, different peers can use different MAC addresses for the server.

Note, modes 0, 1, 2 and, 3 provide fault tolerance and load balancing. Here, we have chosen the active backup mode 1 for the bonded interface. This is because this mode offers redundancy during blocked VLC link and provides load balancing as well as linear scaling of the bandwidth, thus improving the link reliability. To enable Ethernet bonding, IMC is configured with a logical Ethernet BI, which aggregates three physical Ethernet interfaces (PEIs) each connected to LS. In active-backup bonding mode, one of the three Ethernet interfaces acts as 'Active' and the other two as 'Backups'. To monitor VLC connectivity, we use a ARP monitoring technique (at a predefined frequency with a pre-configured ARP target as the switch WAN address) for the logical EBI to supervise which of the three Ethernet links are active at any given time. The reason for this is, closer the ARP target to IMC the faster the round trip outage duration (i.e., from the ARP target via the receiving ARP).

The working principle of ARP monitoring based on active backup Ethernet bonding is as follows. The BI polls the ARP target at a given interval and waits for acknowledgement signal via physically configured Ethernet interfaces. Based on the acknowledgement signal, the logical EBI decides which PEI should be used for transmitting/receiving traffics. The logical EBI uses the 'Active' physical link to send/receive traffic provided the 'Active' link transmits the acknowledgement signal, regardless of ARP reply signals from the 'Backup' link. However, when the 'Active' link does not provide ARP replies, the logical EBI sends/receives traffics via the 'Backup' link provided the 'Backup' link send the ARP replies.

\section{Channel optimization}

In this work, we use a 'better' value in case of failure of the primary slave in the primary-reselect-policy configuration. This means that the best link based on the speed and duplexity is selected continuously while receiving ARP acknowledgments.

However, achieving the best data throughput depends directly on a significant factor of the ARP frequency. In this research work, we use three different ARP frequencies to send ARP requests to the ARP target. Note, there is a trade-off between a channel aware system and higher ARP frequencies. It is obvious that, increasing the number of ARP requests leads to early detection of VLC link failure. However, in a channel aware system increasing the request for ARP will lead to the system overloaded and therefore saturation. As a result, we have path bouncing between the VLC links, which leads to increased $T_{\text {hod }}$ up to several seconds. Therefore, the highest ARP interval value we configure to transmit ARP request is every $50 \mathrm{~ms}$ which has been achieved after observing and analyzing different ARP intervals ranges from 20 to $200 \mathrm{~ms}$.

Fig. 8 presents the view of the interaction between LAP and the $A G V$ client moving from $A_{3}$ to $A_{1}$ passing through

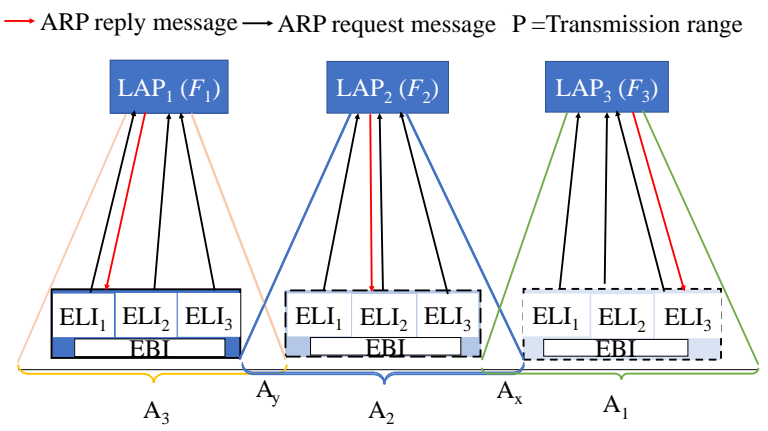

Fig. 8. The view of the interaction between LAP and the AGV client moving from A3 to A1 passing through three LAPs' network coverage.

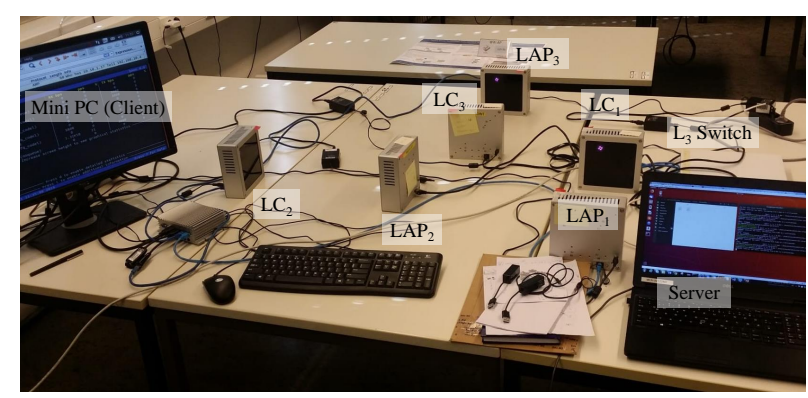

Fig. 9. Experimental indoor VLC setup.

three LAPs' network coverage. At the client system installed on the AGV, Ethernet light interface $1\left(\mathrm{ELI}_{1}\right)$ is assigned with $F_{1}, \mathrm{ELI}_{2}$ is assigned with $F_{2}$ and $\mathrm{ELI}_{3}$ is assigned with $F_{3}$. At $\mathrm{A}_{3}$ position, ARP reply is received only at $\mathrm{ELI}_{1}$, which is used for data communication by Ethernet bond interface (EBI). At $A_{2}$ and $A_{1}$, ARP replies are received at $E_{2 I}$ and $E I_{3}$, respectively; so only the $\mathrm{ELI}_{2}$ and $\mathrm{ELI}_{3}$ are respectively selected for data communication by EBI at $\mathrm{A}_{2}$ and $\mathrm{A}_{1}$ transmission ranges. To enable variable-outage duration handover, at $\mathrm{A}_{y}$, even though ARP replies are received at $E_{1}$ and $E_{2} I_{2}$, the higher priority interface ELI ${ }_{1}$ is used for communications by EBI; similarly, at $\mathrm{A}_{x}$, even though ARP replies are received at $\mathrm{ELI}_{2}$ and $\mathrm{ELI}_{3}$, the higher priority interface $\mathrm{ELI}_{2}$ is used for communication by EBI. Priorities between $\mathrm{ELI}_{1}, \mathrm{ELI}_{2}$ and $\mathrm{ELI}_{3}$ are manually configurable at EBI.

\section{Practical Experiment Results}

The proposed FDLA system is experimentally implemented, and its performance validated. As shown in Fig. 9, we have used 6-LS in three pairs of LCs and LAPs with the distances of 1 and $2 \mathrm{~m}$ between each pair and between each LC and LAP, respectively in a $5 \times 4 \times 2.7 \mathrm{~m}^{3}$ room. A mini- PC with LinuxUbuntu operation system is used as the client and is connected to 3-LC using Ethernet cables to form an IMC system. 2-LAP are connected to a remote server via a configurable switch. LSs are configured using three frequency bands, see Table II. The BI for controlling the traffics over 3-PEI on VLC devices managed using the mini-PC on the client side. In addition, ARP monitoring is configured on top of the BI using Linux 
Netplan tool. Therefore, based on the defined ARP intervals, at any specific time (i) BI sends an ARP broadcast message to everyone with the target as a switch IP address; and (ii) the ARP target receives the packet via the VLC link and determine the MAC address of a specified host by its IP address. , i.e. the host is available and ready to respond to any requests. If the MAC address is correct, then the link is available to transfer the data traffic. In the case of current primary link being lost, the BI looks for second reserved interface. The following subsections present the experimental results for three different traffic types as TCP, UDP and Internet control message protocol (ICMP), which are implemented for real-case scenarios. Note, each test is repeated 10 times per scenario to ensure credibility of results. Moreover, the $T_{\text {hod }}$ values in following subsections figures are shown with a red double link arrow.

\section{A. VLC handover in a linear movement in V-VLC network}

As was described in subsection $\mathrm{V}$-A, this is the most common scenario in $\mathrm{V}-\mathrm{VLC}$, where an AGV moving around in a linear motion will lose connectivity due to fast movements, which results in multiple handovers. With no handover, the maximum $T_{\text {hod }}$ is $15 \mathrm{~s}$, see Fig. 3, compared with $0.2 \mathrm{~s}$ for FDLA.

1) UDP stream: Here, we use Iperf tools to measure the network performance actively. Iperf has two functionalities as the server and client and can create data streams for network's data throughput measurement in one or two directions. The Iperf output is a time-stamped report containing the data (TCP or UDP) transferred and the measured throughput. First, the UDP packets of $1 \mathrm{Mbps}$ is transferred from the remote server to the client using a downlink. We repeat this for three different values of ARP intervals under the same condition to analyze each link's quality and measure $T_{\text {hod }}$ based on the achieved time-stamped. Fig. 10 depicts the average data throughput for a range of ARP intervals of 50, 100 and, $200 \mathrm{~ms}$. As shown, for the ARP interval of $50 \mathrm{~ms}, T_{\text {hod }}$ of value is $0.3 \mathrm{~s}$, see Fig. 10c.

2) TCP stream: Here, we have created the traffic between the client and the remote server and used the uplink for communication between the AGV and the corresponding LAPs. $T_{\text {hod }}$ is measured using the Iperf tool as before for the uplink. One of the most common type of traffic used in V-VLC is the unicast packets. To minimize the packet loss, we have applied FDLA using different ARP frequencies. Fig. 11 presents the average throughput of TCP for ARP intervals of 200, 100 and, $50 \mathrm{~ms}$ for scenario 1 with a single link failure. The measured average $T_{\text {hod }}$ values are $1.5,0.7$ and, 0.3 s, i.e., decreasing with ARP.

3) ICMP stream: To monitor the V-VLC network reliability, we have conducted a set of experiments using the fping network monitoring tool and ICMP, which defines the type of traffic. In fping, several key parameters including the time interval between two pings, number of pings to be send to each host and data in byte can be defined. The data packet size is 100 byte and 500 ICMP packets are transmitted from the client to the remote server every $5 \mathrm{~ms}$. We have measured the interruption time $T_{\text {int }}$ of FDLA for ARP values of 50, 100 and $200 \mathrm{~ms}$ as shown in Fig. 12. Note, $T_{\text {int }}$ is the time difference between

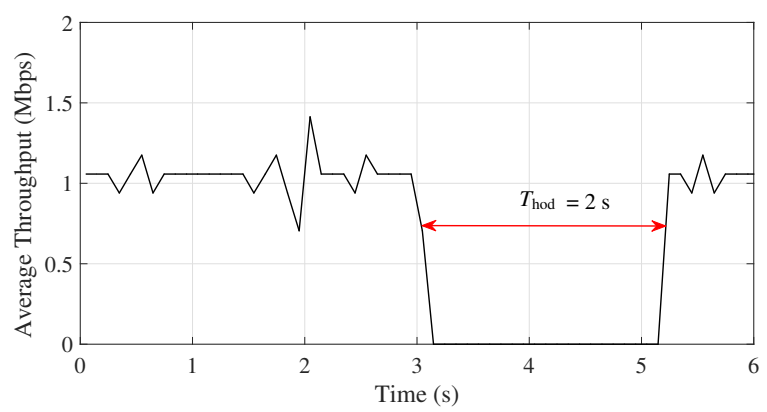

(a)

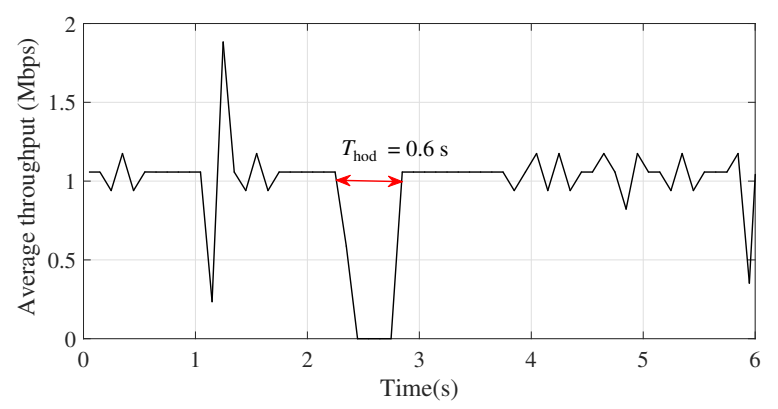

(b)

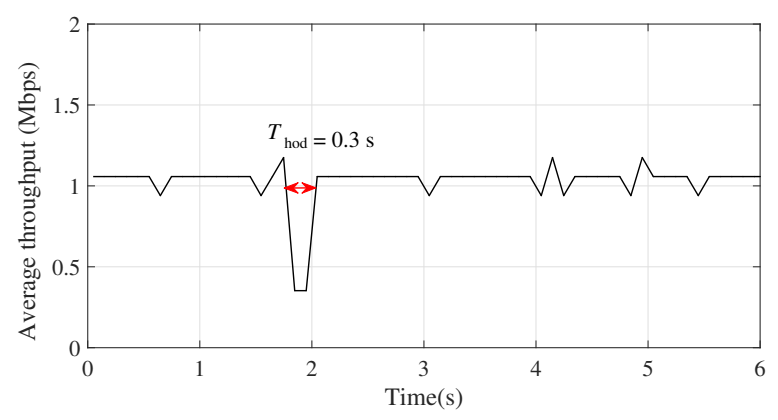

(c)

Fig. 10. The UDP average throughput for ARP intervals of (a) 200, (b) 100 and, (c) $50 \mathrm{~ms}$ for scenario 1 with a single link failure.

the last received ping prior to handover and the first ping request following handover. As shown in Fig. 12, we observe the average value of $T_{\text {int }}$ as $0.72,0.73$, and $2.56 \mathrm{~s}$ for ARP intervals 50, 100, and $200 \mathrm{~ms}$, respectively.

\section{B. VLC handover in V-VLC network: Lose of two VLC connections synchronously}

Here, we emulate and evaluate the extreme case, where the AGV experience two link failures due to blocking, shadowing, or U-turn, synchronously. We analyze the FDLA performance and observe how the V-VLC network performs in this scenario well explained in subsection V-B.

1) UDP stream: Using the Iperf tool we have created and transmitted UDP traffic and use the UDP stream for updating the AGVs continuously with the key information such as AGVs current location, next position, and route path. Note, the V-VLC network should be highly reliable and flexible specially for extreme cases, see subsection V-B. Therefore, we have emulated the scenario and measured the average data throughput for ARP intervals of 200, 100 and $50 \mathrm{~ms}$ 


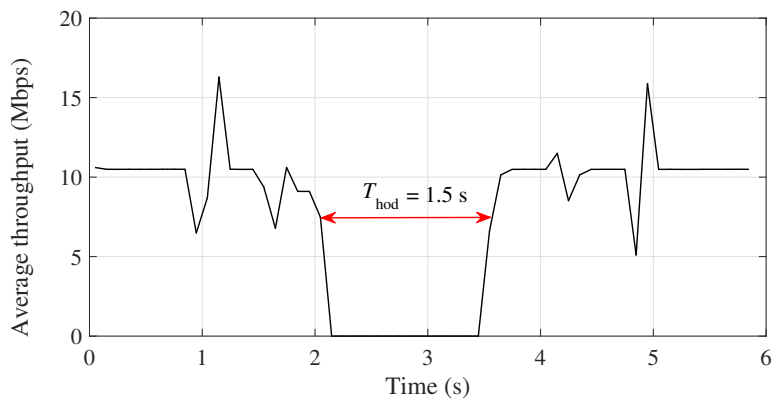

(a)

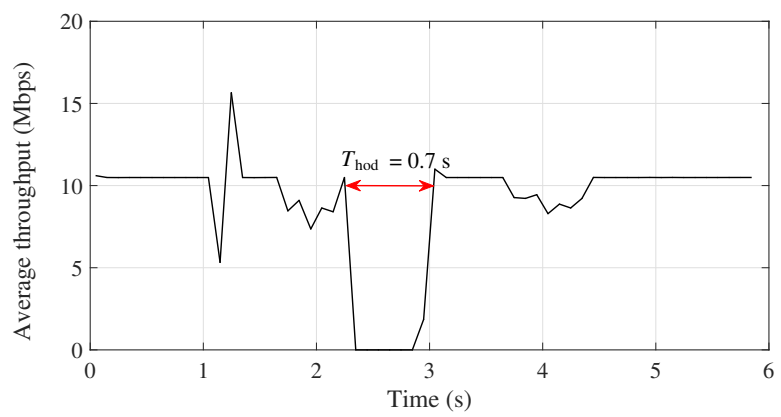

(b)

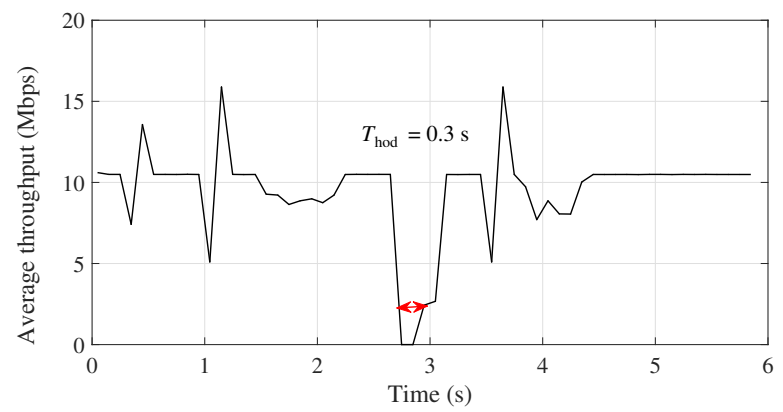

(c)

Fig. 11. TCP Average throughput vs. time for interval (a) 200, (b) 100 and, (c) $50 \mathrm{~ms}$ for scenario 1 with single link broken.

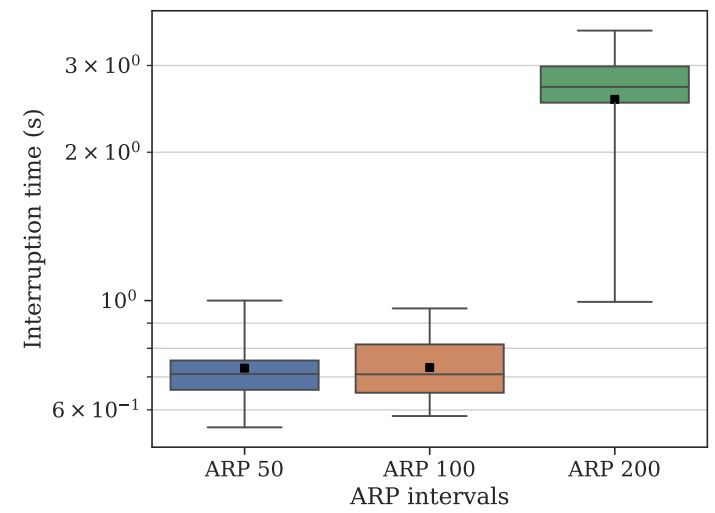

Fig. 12. Interruption time vs. ARP intervals in scenario 1
TABLE III

$T_{\text {hod }}$ VS. ARP INTERVAL VALUES IN SCENARIO 2 , USING TCP AND UDP.

\begin{tabular}{llll}
\hline $\begin{array}{l}\text { ARP interval (ms) } \\
T_{\text {hod }}(s)\end{array}$ & $\mathbf{5 0}$ & $\mathbf{1 0 0}$ & $\mathbf{2 0 0}$ \\
\hline UDP & 0.3 & 0.5 & 2.5 \\
TCP & 0.2 & 0.8 & 3 \\
\hline
\end{tabular}

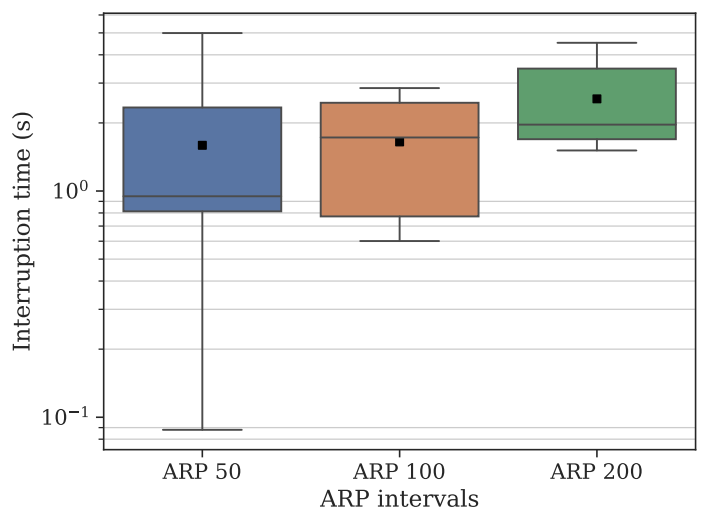

Fig. 13. Interruption time vs. ARP intervals in scenario2

for scenario 2 with two simultaneous links, see Table III. $T_{\text {hod }}$ values shown are 2.5, 0.5 and $0.3 \mathrm{~s}$ for ARP of 200, 100 and, $50 \mathrm{~ms}$, respectively, where the latter offers the best $T_{\text {hod }}$, which is the same as the threshold value given in Fig. 3.

2) TCP stream: To send information to a specific destination we have used the TCP traffic for unicast transmission and evaluated the V-VLC network performance with FDLA. Table III depicts the TCP average throughput for ARP intervals of 200, 100 and $50 \mathrm{~ms}$ for scenario 2 for the case with two simultaneous link failures. As it is shown, the lowest handover delay time of $0.2 \mathrm{~s}$ is achieved for TCP.

3) ICMP stream: Here, we evaluate the VLC network performance using the fping monitoring tool. Note that, for the case of AGV experiencing two simultaneous link failures all failed links are re-connected. 500 ICMP packets are transmitted from the client to the remote server. Fig. 13 depicts $T_{\text {int }}$ as a function of ARP intervals for scenario 2, where the lowest $T_{\mathrm{int}}$ is observed for APR interval of $50 \mathrm{~ms}$. However, the network experiences a large gap between the minimum and maximum interruption times, and this makes the average value stays far from the median number.

TABLE IV

$T_{\text {hod }}$ VS. ARP INTERVAL VALUES IN SCENARIO 3 , USING TCP AND UDP.

\begin{tabular}{lcccccc}
\hline & \multicolumn{3}{c}{ Handover $\mathbf{1}$} & \multicolumn{3}{c}{ Handover } \\
ARP interval (ms) & $\mathbf{5 0}$ & $\mathbf{1 0 0}$ & $\mathbf{2 0 0}$ & $\mathbf{5 0}$ & $\mathbf{1 0 0}$ & $\mathbf{2 0 0}$ \\
$T_{\text {hod }}(s)$ & & & & & & \\
\hline UDP & 0.3 & 0.5 & 2.5 & 0.3 & 0.4 & 1.6 \\
TCP & 0.4 & 0.6 & 1.55 & 0.3 & 0.8 & 1.7 \\
\hline
\end{tabular}


TABLE V

NUMBER OF THE PACKET DELIVERY VS. ARP INTERVAL VALUES IN THE 3 SCENARIOS.

\begin{tabular}{llllllllll}
\hline $\begin{array}{l}\text { Statistics } \\
\text { Packet Delivery percentage }\end{array}$ & ARP 50 & $\begin{array}{c}\text { Scenario 1 } \\
\text { ARP 100 }\end{array}$ & ARP 200 & ARP 50 & $\begin{array}{l}\text { Scenario 2 } \\
\text { ARP 100 }\end{array}$ & ARP 200 & ARP 50 & $\begin{array}{l}\text { Scenario 3 } \\
\text { ARP 100 }\end{array}$ & ARP 200 \\
\hline Average (\%) & 99.8 & 99.8 & 99.44 & 99.62 & 99.76 & 99.44 & 99.64 & 99.36 & 98.86 \\
Minimum (\%) & 99.8 & 99.8 & 99.2 & 99 & 99.4 & 99 & 99.2 & 98.2 & 98 \\
Maximum (\%) & 99.8 & 99.8 & 99.8 & 100 & 99.8 & 99.6 & 99.8 & 99.6 & 99.2 \\
\hline
\end{tabular}

C. VLC handover in a two dimensions V-VLC network: Lose of two VLC connections asynchronously

Here, we emulate and evaluate the scenario where AGV can move between the defined parallel paths, see subsection V-C. In this case, there is a very high possibility for AGV to lose its connectivity with the reachable LAPs.

1) UDP stream: In this experimental setup, we analyze how FDLA achieves the network connection following each handover between LAPs. This might be due to AGV not being able to utilize the two VLC links when making a a U-turn or experiencing shadowing/blocking. Here, we consider two VVLC network handovers as presented in Table IV. Transmitting UDP, we achieve the shortest handover delay as $0.3 \mathrm{~s}$ during first and second handovers, once ARP interval is configured as $50 \mathrm{~ms}$, respectively. The use of ARP interval value of 100 ms results in achieving $T_{\text {hod }}$ values of 0.4 and $0.5 \mathrm{~s}$ with two handovers.

2) TCP stream: We measure and analyze $T_{\text {hod }}$ once AGV transfers unicast packets to the remote server. The client starts transferring TCP packets to a destination as the remote server and meanwhile at $T=t_{1}$ and $t_{2}$, two VLC link connections break and the BI which is installed on the client machine, immediately re-establishes the link with the third available link on the LAP on the third frequency range. Table IV presents $T_{\text {hod }}$ using the TCP packet transmission for three different ARP interval values. Based on how fast BI transmits the ARP request, it detects the failed link and establishes a new connection. Therefore, in ARP interval 200 and $100 \mathrm{~ms}$ the number of the packet lost increases due to not frequent ARP request transmission. However, for the ARP interval of $50 \mathrm{~ms}, T_{\text {hod }}$ is within the range of 0.3 to $0.4 \mathrm{~s}$ since the channel is monitored more often.

3) ICMP stream: Here, we analyze FDLA reliability performance and measure the total packet delivery for transmitting 500 ICMP packet every $5 \mathrm{~ms}$. Table $\mathrm{V}$ presents minimum, maximum, and average packet delivery in a network for three scenarios. As shown, in scenarios 2 and 3 with two failed VLC links, FDLA offers the highest packet delivery of $99.5 \%$.

\section{CONCLUSION}

This paper presented a handover solution known as FDLA in Data link layer based on the frequency diversity and the link aggregation for the V-VLC networks. FDLA used link aggregation techniques over VLC Data link layer to provide an efficient channel selection method while using multiple VLC channels with different frequencies, thus ensuring smooth handover with minimum delay by detecting the failed links and establishing new links within a short time period. Moreover,
FDLA addressed the channel selection problem among multiple bonded interfaces by selecting the best available link based on speed and duplexity. We considered three most common scenarios by means of practical implementation, where AGV experienced one or more links failures. The experimental results showed that, the handover outage duration time reduced by orders of magnitude for FDLA when using different ARP intervals compared with methods using no handover technique. The future works will focus on real mobility network scenarios extending the FDLA prototype and introducing a new channel selection technique where also other features can be considered.

\section{REFERENCES}

[1] P. H. Pathak, X. Feng, P. Hu, and P. Mohapatra, "Visible Light Communication, Networking, and Sensing: A Survey, Potential and Challenges," IEEE Communications Surveys \& Tutorials, vol. 17, no. 4, pp. 2047 - 2077, Feb. 2015

[2] C. Sommer, R. German, and F. Dressler, "Bidirectionally Coupled Network and Road Traffic Simulation for Improved IVC Analysis," IEEE Transactions on Mobile Computing (TMC), vol. 10, no. 1, pp. 3-15, Jan. 2011.

[3] D. Miras, L. Maret, M. Maman, M. Laugeois, X. Popon, and D. Ktenas, "A high data rate $\mathrm{LiFi}$ integrated system with inter-cell interference management," in IEEE Wireless Communications and Networking Conference (WCNC 2018). Barcelona, Spain: IEEE, Apr. 2018.

[4] L. U. Khan, "Visible light communication: Applications, architecture, standardization and research challenges," Digital Communications and Networks, vol. 3, no. 2, pp. 78-88, May 2017.

[5] E.-J. Kim, J.-H. Kwon, D. Kim, and Y. Lim, "Distributed Interferenceaware Medium Access Control for IEEE 802.15.7 Visible Light Communications," Sensors and Materials, vol. 30, no. 8, p. 1665, Aug. 2018.

[6] Q. Mao, P. Yue, M. Xu, Y. Ji, and Z. Cui, "OCTMAC: A VLC based MAC protocol combining optical CDMA with TDMA for VANETs," in IEEE International Conference on Computer, Information and Telecommunication Systems (CITS 2017). Dalian, China: IEEE, Jul. 2017.

[7] Q. Wang, D. Giustiniano, and M. Zuniga, "In Light and In Darkness, In Motion and In Stillness: A Reliable and Adaptive Receiver for the Internet of Lights," IEEE Journal on Selected Areas in Communications (JSAC), vol. 36, no. 10, pp. 149-161, Oct. 2018

[8] E. Alizadeh Jarchlo, S. M. Kouhini, H. Doroud, G. Maierbacher, M. Jung, B. Siessegger, Z. Ghassemlooy, A. Zubow, and G. Caire, "Flight: A Flexible Light Communications network architecture for indoor environments," in 15th International Conference on Telecommunications (ConTEL 2019). Graz, Austria: IEEE, Jul. 2019.

[9] E. Dinc, O. Ergul, and O. B. Akan, "Soft Handover in OFDMA Based Visible Light Communication Networks," in 82nd IEEE Vehicular Technology Conference (VTC 2015-Fall). Boston, MA: IEEE, Sep. 2015.

[10] J. Xiong, Z. Huang, Y. Ji, and K. Zhuang, "A cooperative positioning with Kalman filters and handover mechanism for indoor microcellular visible light communication network," Optical Review, vol. 23, no. 4, pp. 683-688, May 2016.

[11] E. Alizadeh Jarchlo, S. M. Kouhini, H. Doroud, E. Eso, P. Gawłowicz, M. Zhang, B. Siessegger, M. Jung, Z. Ghassemlooy, G. Caire, and A. Zubow, "Analyzing interface bonding schemes for VLC with mobility and shadowing," in 12th IEEE/IET International Symposium on Communication Systems, Networks and Digital Signal Processing (CSNDSP 2020). Virtual Conference: IEEE, 72020. 
[12] E. Alizadeh Jarchlo, P. Gawłowicz, H. Doroud, B. Siessegger, M. Jung, G. Caire, A. Zubow, and Z. Ghassemlooy, "A Flexible Transport Layer Protocol Architecture for Handover in a Vehicular VLC Network," in 12th IEEE/IET International Symposium on Communication Systems, Networks and Digital Signal Processing (CSNDSP 2020). Virtual Conference: IEEE, 72020.

[13] A. M. Vegni and T. D. C. Little, "Handover in VLC systems with cooperating mobile devices," in 8th IEEE International Conference on Wireless and Mobile Computing, Networking and Communications (ICNC 2012). Maui, HI: IEEE, Oct. 2012.

[14] D. Wu, Z. Ghassemlooy, W. Zhong, and C. Chen, "Cellular indoor OWC systems with an optimal lambertian order and a handover algorithm," in 7th International Symposium on Telecommunications (IST 2014). Tehran, Iran: IEEE, Sep. 2014.

[15] M. Rahaim and T. D. C. Little, "Toward practical integration of dual-use VLC within 5G networks," IEEE Wireless Communications, vol. 22, no. 2, pp. $97-103$, Apr. 2015.

[16] M. Hammouda, J. Peissig, and A. M. Vegni, "Design of a cognitive VLC network with illumination and handover requirements," in IEEE International Conference on Communications (ICC 2017), 3rd Workshop on Optical Wireless Communications (OWC 2017). Paris, France: IEEE, May 2017.

[17] A. Zubow, S. Zehl, and A. Wolisz, "BIGAP - Seamless handover in high performance enterprise IEEE 802.11 networks," in IEEE/IFIP Network Operations and Management Symposium (NOMS 2016). Istanbul, Turkey: IEEE, Apr. 2016.

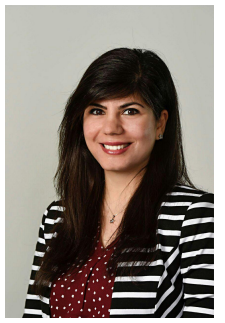

Elnaz Alizadeh Jarchlo Elnaz Alizadeh Jarchlo received the B.s in Software Engineering degree in 2008 and the M.s in Information system degree in 2013 from Azad University and Middle East Technical University, respectively. She has participated in several European and international projects in universities and research institutes such as UGent, iMinds, IMDEA Netowkrs, University Carlos III of Madrid, University of Chinese Academy of Sciences and Fraunhofer Institute for Telecommunications (HHI) She has published a number of journal/conference papers and a book chapter. Her interests are in the field of MAC and upper layer protocols for wireless communications and visible light communications. She is currently a $\mathrm{PhD}$ candidate at TU Berlin, working on VisIoN project, a Marie Sklodowska-Curie Innovative Training Network (MSCA ITN)program.

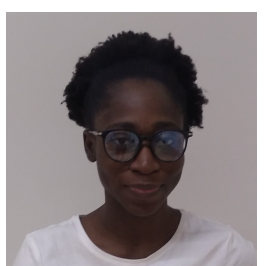

Elizabeth Eso Elizabeth Eso received her B.Eng in Electrical and Electronics Engineering from the Abubakar Tafawa Balewa University, Bauchi, Nigeria in 2011 and MSc in Microelectronics and Communications Engineering from the Northumbria University Newcastle, UK in 2014. She was the second prize winner in the IEEE students project presentation competition in the North East Region Network, UK in 2014. She joined the Federal University of Technology, Akure, Nigeria as an academic staff in 2016. She is currently a $\mathrm{PhD}$ researcher with the Optical Communications Research Group, Northumbria University Newcastle, UK, under the prestigious Marie Sklodowska-Curie Actions. She has published a number of journal and conference papers. She is a member of IEEE and IET. Her research interest include visible light communications for vehicular applications, optical camera communications and free space optics.



Hossein Doroud Hossein Doroud graduated from the B.s in Electrical Engineering degree in 2009 from Central Tehran Branch of Islamic Azad University. Following he succeeds to received the M.s and $\mathrm{Ph} . \mathrm{D}$. in Telematics Engineering degree in 2013 and 2019 respectively from Universidad Carlos III de Madrid University. He has been hosted by several international research groups and institutes such as COMICS, IMDEA Network, and TKN - TU Berlin as a researcher visitor. He has published several journal/conference papers. His interests are in the field of Network and Internet measurement, Network Security, and Visible Light Communication. He is currently a Postcode researcher in the Telecommunication Networks Group at TU Berlin.



Anatolij Zubow Anatolij Zubow is a senior researcher at the Telecommunication Networks (TKN) group. His research interests are in architectures and protocols of wireless communication networks as well as in protocol engineering with impact on performance and QoS aspects. Recently he is focusing mainly on coexistence of heterogeneous wireless technologies in unlicensed spectrum, highperformance IEEE 802.11 networks, software-defined wireless networking and ultra-reliable low latency communication. He has strong interest in prototyping experimental work and testbeds. In the past, he did research in the area of wireless ad-hoc mesh networks at the Humboldt Universität zu Berlin where he received his $\mathrm{PhD}$ degree in 2009. Moreover, he had two research visits undertaken at the NEC Network Laboratories in Heidelberg, where he was working on future mobile networks.

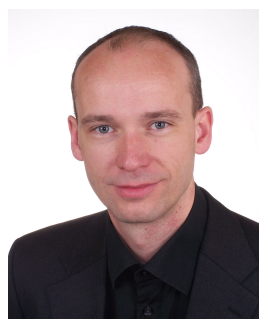

Falko Dressler (dressler@ccs-labs.org) Falko Dressler is full professor and Chair for Data Communications and Networking at the School of Electrical Engineering and Computer Science, TU Berlin. He received his M.Sc. and Ph.D. degrees from the Dept. of Computer Science, University of Erlangen in 1998 and 2003, respectively. Dr. Dressler has been associate editor-in-chief for IEEE Trans. on Mobile Computing and Elsevier Computer Communications as well as an editor for journals such as IEEE/ACM Trans. on Networking, IEEE Trans. on Network Science and Engineering, Elsevier Ad Hoc Networks, and Elsevier Nano Communication Networks. He has been chairing conferences such as IEEE INFOCOM, ACM MobiSys, ACM MobiHoc, IEEE VNC, IEEE GLOBECOM $\mathrm{He}$ authored the textbooks Self-Organization in Sensor and Actor Networks published by Wiley \& Sons and Vehicular Networking published by Cambridge University Press. He has been an IEEE Distinguished Lecturer as well as an ACM Distinguished Speaker. Dr. Dressler is an IEEE Fellow as well as an ACM Distinguished Member. He is a member of the German National Academy of Science and Engineering (acatech). He has been serving on the IEEE COMSOC Conference Council and the ACM SIGMOBILE Executive Committee. His research objectives include adaptive wireless networking (radio, visible light, molecular communications) and embedded system design (from microcontroller to Linux kernel) with applications in ad hoc and sensor networks, the Internet of Things, and cooperative autonomous driving systems. 


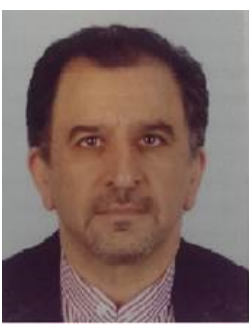

Zabih Ghassemlooy Prof. Zabih Ghassemlooy FOSA, FIET, SMIEEE: BSc (Hons.) in EE Engineering, Manchester Metropolitan Univ., (1981), MSc (1984) and PhD (1987) from Manchester Univ., UK. 1987-88 he was a Post-Doctoral Research Fellow at City Univ., UK. 2004-14 joined Faculty of Eng. \& Env., Northumbria Univ. as an Associate Dean Research, and currently is the Head of Optical Communications Research Group. He is a Research Fellow (2016) and a Distinguished Professor (2015) at the Chinese Academy of Science. He has over 900 publications (with more than 350 in Journals). He has published over 900 papers (more than 350 journals and 8 books), 100 keynote/invited talks and supervised 10 Research Fellows and 65 PhDs. His research interests are in the areas of optical wireless communications, free space optics, visible light communications, hybrid RF and optical wireless communications. He is the Chief Editor of the British Journal of Applied Science and Technology and the International Journal of Optics and Applications, Associate Editor of a number of international journals, and Co-guest Editor of a number of special issues. He is the Vice-Cahir of OSA Technical Group of Optics in Digital Systems (2018-). He is the Chair of the IEEE Student Branch at Northumbria University, Newcastle (2019-). From 2004-06 he was the IEEE UK/IR Communications Chapter Secretary, the Vice-Chairman (2006-2008), the Chairman (2008-2011), and Chairman of the IET Northumbria Network (Oct 2011-2015).

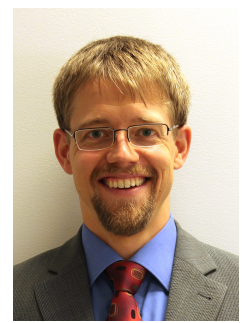

Bernhard Siessegger Bernhard Siessegger received his Diploma in EE from University of Ulm and earned his doctoral degree (Dr.-Ing.) from the Technical University Dresden in 2004. He was awarded the Heinrich Barkhausen prize for his thesis donated by the Carl Friedrich von Siemens foundation, nominated by the faculty of Electrical and Computer Engineering of the TU Dresden. He then joined OSRAM as a project and development engineer. $\mathrm{He}$ also worked as an electronics specialist on technology evaluation. In 2010 he became Manager of Research at the OSRAM research facility in Beverly, Mass. in the US. From 2012 on he was leading the global Technology Field Electronics within OSRAM Corporate Innovation, with operations in Germany as well as the US. Major activities of the team were in the area of sensors and communications, including Indoor localization and Wi-Fi \& LiFi from the luminaire. His work was honored by the OSRAM Orange Award, the OSRAM-internal "OSCAR" in the category "Employee Excellence." In 2019 he was appointed Senior Principal Key Expert heading the global Core Technology Field "Power Electronics, Controls \& Connectivity" in OSRAM. He is currently serving as Technology Lead and CTO of VISN (www.visn.io). VISN is an OSRAM-internal startup offering services in smart buildings utilizing privacy-preserving occupancy and activity recognition technologies. He holds more than 100 granted patents in the area of lighting, electronics, sensors and communication.

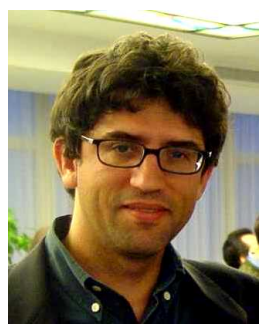

Giuseppe Caire Giuseppe Caire (S '92 - M '94 SM '03 - F' 05 ) was born in Torino in 1965 . He received the B.Sc. in Electrical Engineering from Politecnico di Torino in 1990, the M.Sc. in Electrical Engineering from Princeton University in 1992, and the Ph.D. from Politecnico di Torino in 1994. He has been a post-doctoral research fellow with the European Space Agency (ESTEC, Noordwijk, The Netherlands) in 1994-1995, Assistant Professor in Telecommunications at the Politecnico di Torino, Associate Professor at the University of Parma, Italy, Professor with the Department of Mobile Communications at the Eurecom Institute, Sophia-Antipolis, France, a Professor of Electrical Engineering with the Viterbi School of Engineering, University of Southern California, Los Angeles, and he is currently an Alexander von Humboldt Professor with the Faculty of Electrical Engineering and Computer Science at the Technical University of Berlin, Germany.

He received the Jack Neubauer Best System Paper Award from the IEEE Vehicular Technology Society in 2003, the IEEE Communications Society \& Information Theory Society Joint Paper Award in 2004 and in 2011, the Leonard G. Abraham Prize for best IEEE JSAC paper in 2019, the Okawa Research Award in 2006, the Alexander von Humboldt Professorship in 2014 the Vodafone Innovation Prize in 2015, and an ERC Advanced Grant in 2018 Giuseppe Caire is a Fellow of IEEE since 2005. He has served in the Board of Governors of the IEEE Information Theory Society from 2004 to 2007, and as officer from 2008 to 2013. He was President of the IEEE Information Theory Society in 2011. His main research interests are in the field of communications theory, information theory, channel and source coding with particular focus on wireless communications. 